\title{
Caractéristiques radiologiques des canaux de transport d'eau - Exemple du réseau hydraulique régional Languedoc Roussillon
}

\author{
F. Eyrolle-Boyer ${ }^{1 \text { a }}$, Ph. Renaud ${ }^{1}$, F. Le Dore ${ }^{2}$, D. Tournieux ${ }^{1}$, D. Claval ${ }^{1}$, J.-F. Blanchet ${ }^{2}$, \\ C. Antonelli ${ }^{1}$, M. Zebracki ${ }^{1}$, C. Cossonnet ${ }^{1}$, B. Boulet ${ }^{1}$, X. Cagnat ${ }^{1}$, A. Devisme ${ }^{1}$ et R. Gurriaran ${ }^{1}$ \\ 1 IRSN, PRP-ENV/SESURE/LS3E, STEME/LMRE, SESURE/LERCM, BP 3, 13115 Saint Paul Lez Durance, France. \\ 2 BRL Direction Générale, 1105 avenue Pierre Mendès France, BP 94001, 30001 Nîmes Cedex 5, France.
}

Reçu le 8 avril 2013 - Accepté le 18 octobre 2013

\begin{abstract}
Résumé - Une étude radioécologique a été menée sur les canaux rhodaniens du réseau hydraulique régional propriété de la région Languedoc Roussillon, gérés par BRL. Ces canaux transfèrent de l'eau du fleuve Rhône vers les territoires des départements du Gard et de l'Hérault à des fins d'irrigation et de production d'eau potable. Nos résultats montrent que les caractéristiques hydrauliques intrinsèques des canaux de transport d'eau influent sur la distribution solide/solution des éléments traces en transit et par conséquent sur leur transfert vers les milieux récepteurs. Si les concentrations en phase dissoute (eau filtrée) sont conservées, les concentrations en phase particulaire (matières en suspension et sédiments) sont significativement modifiées au cours du transit. Outre la ségrégation granulométrique des particules entre l'amont et l'aval du système, ces résultats sont très probablement liés à la production biologique autochtone (phyto et zooplancton). Ces résultats originaux soulignent le caractère atypique des canaux de transport d'eau quant au transfert des éléments potentiellement contaminants.
\end{abstract}

\begin{abstract}
Radiological characteristics of water transport canals - Example of the Rhone Languedoc Roussillon regional network. In many places in the world, canals represent a significant cumulative linear effect on the flow of surface waters and play a significant role in the redistribution of water resources. A radioecological study was conducted on the Languedoc Roussillon regional Rhodanian canals which transfer water from the river Rhône to the territories of the Gard and Herault for irrigation and drinking water supply. Our results show that the hydrological characteristics of water canals may impact significantly on the solid/solution distribution of trace elements in transit, and consequently on their transfer to the receiving environment. In addition to particle size segregation during transit, these results are most likely to be related to biological production (phytoplankton and zooplankton). These results highlight that the origin and quality of naturally occurring solid particles could play a fundamental role in the transfer of trace pollutants, especially in irrigation canals.
\end{abstract}

Keywords: Water transport canals / Rhone river / water resources / biological production / naturally occurring particles

\section{Introduction}

En France métropolitaine, près de $40000 \mathrm{~km}$ de canaux servant à la navigation et à l'irrigation sillonnent le paysage. Sur les $8500 \mathrm{~km}$ de voies d'eau navigables, on estime que $4300 \mathrm{~km}$ sont des canaux. Les canaux d'irrigation desservent plusieurs centaines de milliers d'hectares de terrains agricoles dont $80 \%$ sont situés dans le Sud-Est de la France. Un canal de transport d'eau présente des différences hydrauliques notables par rapport à une rivière, même canalisée. En effet les

\footnotetext{
a frederique.eyrolle-boyer@irsn.fr
}

dimensions et le débit de la rivière augmentent de l'amont vers l'aval en raison des apports naturels de ses affluents. Le canal d'irrigation a un débit et un gabarit qui diminuent de l'amont vers l'aval du fait des prélèvements des divers usagers.

Depuis 1960, les canaux rhodaniens du Languedoc Roussillon transfèrent de l'eau du fleuve Rhône vers les départements du Gard et de l'Hérault. L'eau est utilisée aussi bien pour les besoins d'arrosage que pour la production d'eau potable et divers usages industriels, en complément des ressources préexistantes. Aujourd'hui le projet d'extension Aqua Domitia permettra d'étendre ce réseau sur une centaine de communes. Il consiste à apporter une deuxième ressource 


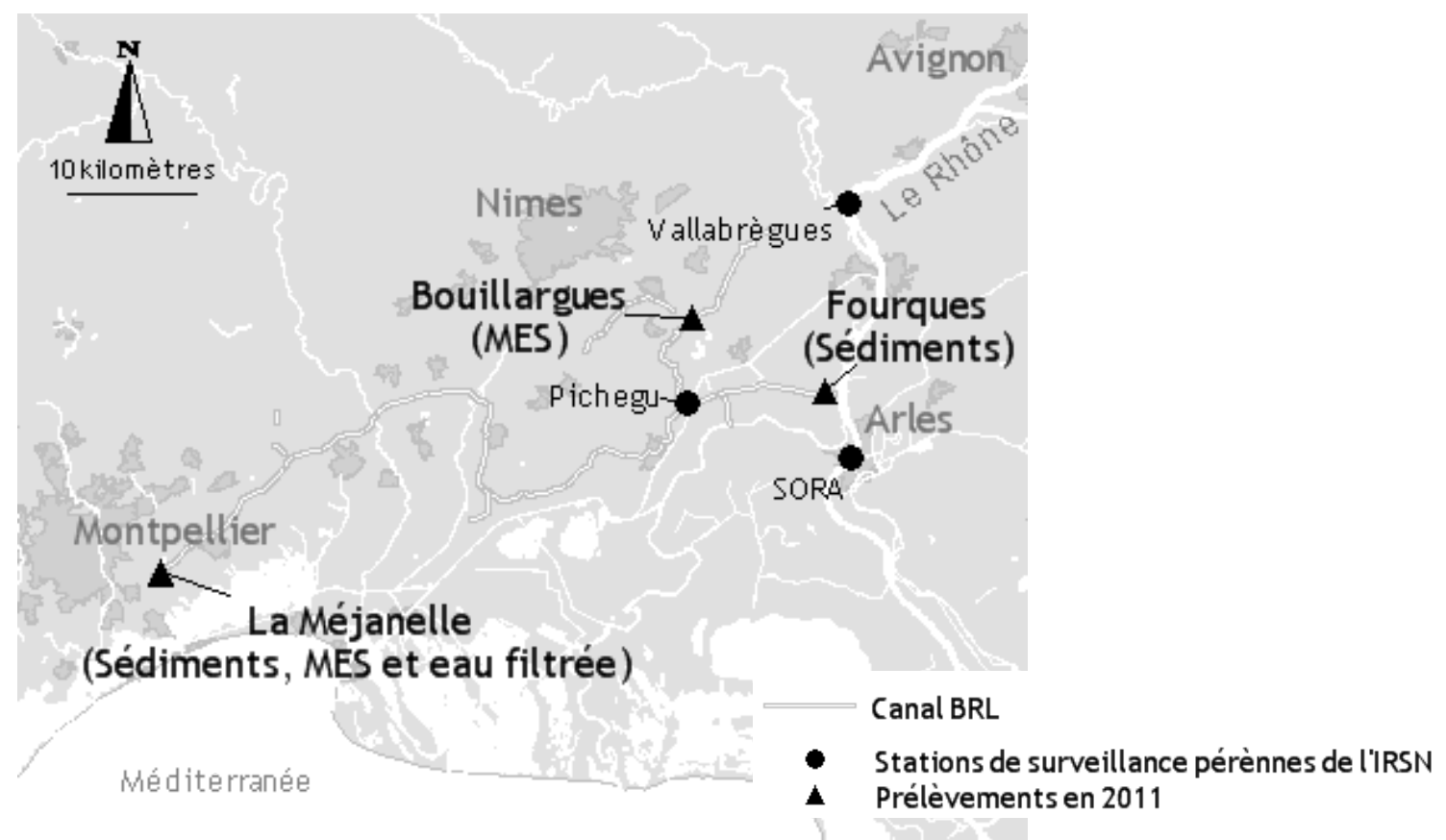

Fig. 1. Localisation des points de suivi et de prélèvements dans le Rhône et les canaux du réseau hydraulique régional propriété de la région Languedoc Roussillon.

Location of monitoring and sampling points in the Rhone river and the regional hydraulic canal network owned by the Region of Languedoc Roussillon.

en eau aux territoires situés dans l'Ouest du département de l'Hérault et dans l'Aude, en maillant les réseaux alimentés par le Rhône avec ceux alimentés par les fleuves côtiers tels que l'Orb, l'Hérault ou l'Aude. La qualité des eaux transférées est au cœur des préoccupations sociétales, ainsi BRL et l'IRSN ont conduit entre 1995 et 2000 trois études destinées à caractériser la radioactivité de l'eau distribuée par le canal, connaître les transferts des radionucléides aux cultures irriguées et l'effet du traitement de potabilisation sur les concentrations en radionucléides de l'eau destinée à la consommation humaine (Jourd'heuil et Lambretchts, 1995; Jourd'heuil et al., 1997). En 2011, une nouvelle étude a été conduite afin de mettre à jour les connaissances sur l'état radiologique des eaux transférées par le canal. Les résultats présentés ici fournissent un comparatif entre les eaux du fleuve et les eaux du canal ainsi que les données acquises en 2011. Nos résultats permettent de statuer sur l'état radiologique du canal aujourd'hui, et plus particulièrement de souligner les particularités des canaux de transport d'eau quant au transfert des polluants.

\section{Matériel et méthode}

\subsection{Surveillance de la radioactivité des eaux du Rhône}

La radioactivité de l'eau du Rhône fait l'objet d'une surveillance depuis les années 60 qui porte à la fois sur les éléments dissous au travers d'analyses de l'eau filtrée et sur la fraction particulaire par l'analyse de boues de décantation ou de matières en suspension (MES). L'une des stations sur le Rhône, se trouve à Vallabrègues, à une vingtaine de kilomètres en amont de la prise d'eau du canal. Les prélèvements y sont effectués au moyen d'un hydro collecteur qui permet de constituer mensuellement un échantillon composite d'eau filtrée et de boues de décantation. En aval, la Station Observatoire du Rhône en Arles (SORA), permet depuis 2005 de mesurer des radionucléides artificiels et naturels présents à l'état de traces dans l'eau filtrée du Rhône et les MES (Eyrolle et al., 2010; Antonelli et al., 2012).

\subsection{Prélèvements et analyses}

Afin d'évaluer l'état radiologique des eaux du canal les différentes matrices (sédiments, MES et eau filtrée) ont été échantillonnées. Les sédiments superficiels ont été prélevés dans le canal d'amenée au niveau de Fourques (Pont de Belleval), à $2 \mathrm{~km}$ de l'entrée du canal (prise d'eau au Rhône), et dans le canal Philippe Lamour au niveau de la station de Pompage de la Méjanelle au Sud-Ouest de Montpellier, à $69 \mathrm{~km}$ de la prise d'eau au Rhône. Trois prélèvements d'eau ont été réalisés sur les canaux. L'un au niveau de la prise gravitaire de la station de traitement de Bouillargues sur le canal de Campagne au Sud-Ouest de Nîmes, les deux autres au niveau de la station de pompage de la Méjanelle. Le tableau 1 et la figure 1 synthétisent la localisation et les caractéristiques des échantillons étudiés ainsi que les analyses effectuées. Les méthodes de traitement et de conditionnement des échantillons pour analyses sont décrites par ailleurs (Eyrolle et al., 2012). 
Tableau 1. Prélèvements et analyses effectués en 2011 au sein du canal géré par BRL. Sampling and analyses conducted in 2011 within the canal managed by BRL.

\begin{tabular}{|c|c|c|c|c|}
\hline \multicolumn{5}{|c|}{ Canal BRL } \\
\hline Date & Localisation & $\begin{array}{l}\text { Distance / } \\
\text { entrée du } \\
\text { canal }(\mathrm{km})\end{array}$ & Type & Analyses \\
\hline 22/07/2011 & $\begin{array}{l}\text { Fourques, } \\
\text { Pont de } \\
\text { Belleval }\end{array}$ & 2 & Sédiments & $\begin{array}{l}\text { Bêta global, alpha global, spectrométrie alpha (isotopes Pu et Am), } \\
{ }^{90} \mathrm{Sr} \text {, TOL, spectrométrie gamma, ICPMS (isotopes U et Th), } \\
\text { analyses granulométriques, matière organique, carbone organique }\end{array}$ \\
\hline $22 / 07 / 2011$ & $\begin{array}{l}\text { La } \\
\text { Méjanelle }\end{array}$ & 69 & Sédiments & $\begin{array}{l}\text { Bêta global, alpha global, spectrométrie alpha (isotopes Pu et Am), } \\
{ }^{90} \mathrm{Sr} \text {, TOL, spectrométrie gamma, ICPMS (isotopes U et Th), } \\
\text { analyses granulométriques, matière organique, carbone organique }\end{array}$ \\
\hline $27 / 07 / 2011$ & $\begin{array}{c}\text { Prise de } \\
\text { Bouillargues }\end{array}$ & 21 & MES & $\begin{array}{l}\text { Spectrométrie alpha (isotopes } \mathrm{Pu} \text { et } \mathrm{Am} \text { ), }{ }^{90} \mathrm{Sr} \text {, spectrométrie } \\
\text { gamma, ICPMS (isotopes } \mathrm{U} \text { et } \mathrm{Th} \text { ), matière organique }\end{array}$ \\
\hline $27 / 07 / 2011$ & $\begin{array}{c}\text { La } \\
\text { Méjanelle }\end{array}$ & 69 & MES & $\begin{array}{c}\text { Spectrométrie alpha (isotopes } \mathrm{Pu} \text { et } \mathrm{Am} \text { ), }{ }^{90} \mathrm{Sr} \text {, spectrométrie } \\
\text { gamma, ICPMS (isotopes } \mathrm{U} \text { et } \mathrm{Th} \text { ), matière organique }\end{array}$ \\
\hline 08/12/2011 & $\begin{array}{l}\text { La } \\
\text { Méjanelle }\end{array}$ & 69 & MES & $\begin{array}{c}\text { Spectrométrie alpha (isotopes } \mathrm{Pu} \text { et } \mathrm{Am}),{ }^{90} \mathrm{Sr} \text {, spectrométrie } \\
\text { gamma, ICPMS (isotopes U et Th), matière organique }\end{array}$ \\
\hline 08/12/2011 & $\begin{array}{l}\text { La } \\
\text { Méjanelle }\end{array}$ & 69 & Eau filtrée & $\begin{array}{l}\text { Alpha global, bêta global, bêta global résiduel, DTI, } \\
\text { spectrométrie alpha (isotopes Pu et Am), }{ }^{90} \mathrm{Sr},{ }^{210} \mathrm{Po} \text {, tritium } \\
\left({ }^{3} \mathrm{H}\right) \text {, spectrométrie gamma, ICPMS (isotopes U et Th), potassium }\end{array}$ \\
\hline
\end{tabular}

\section{Résultats et discussion}

\subsection{Radioactivité des eaux du canal en comparaison avec les eaux du Rhône}

Le canal Philippe Lamour, au niveau de la station de pompage Aristide Dumont (lieu-dit Pichegu Bellegarde 30), a fait l'objet de 1982 à 1997, d'une surveillance analogue à celle effectuée à Vallabrègues. Une étude réalisée en 1995-1996 par l'IPSN comportait également des analyses d'eau filtrée, de matières en suspension (MES) et de sédiments (Jourd'heuil et Lambretchts, 1995 ; Jourd'heuil et al., 1997). Ces éléments permettent de comparer les niveaux de radioactivité de l'eau du Rhône à ceux du canal, aussi bien sur la fraction dissoute que particulaire.

Les activités volumiques en ${ }^{3} \mathrm{H}$ des eaux filtrées du Rhône et du canal sont comme attendu extrêmement proches. Les mêmes observations sont faites pour le ${ }^{90} \mathrm{Sr}$ (Fig. 2) ainsi que pour les ${ }^{106} \mathrm{Ru}$ et ${ }^{125} \mathrm{Sb}$ (non représentés). Ainsi, dans le contexte actuel, la surveillance effectuée sur le Rhône, notamment à Vallabrègues ou à Arles, vaut comme surveillance de l'eau filtrée distribuée par le canal.

En ce qui concerne la fraction particulaire, les activités massiques mesurées dans le canal sont le plus souvent supérieures à celles du Rhône (Fig. 3). Cela est observé pour le ${ }^{134} \mathrm{Cs}$ et le ${ }^{137} \mathrm{Cs}$, le ${ }^{60} \mathrm{Co}$, le ${ }^{238} \mathrm{Pu}$, le ${ }^{239+240} \mathrm{Pu}$ et $\mathrm{l}^{241} \mathrm{Am}$, c'est-à-dire les éléments qui ont une forte affinité avec les particules.

Les MES résiduelles au sein du canal sont plus fines que celles transportées par le Rhône et plus homogènes en taille en lien avec l'hydrologie spécifique du canal. L'activité massique des MES est toujours plus élevée pour les particules les plus fines en raison de la surface d'échange qui est, pour une masse identique de matière de même origine, d'autant plus importante que la taille des particules est petite. La gamme de variabilité des activités massiques au sein du canal est moindre que celle observée dans le fleuve. Cette observation s'explique par une plus grande homogénéité de la taille des particules au sein du canal par rapport au fleuve. En effet, les flux d'eau et de matière en transit dans le fleuve et alimentant le canal ne sont pas conservés de manière proportionnelle. En dehors des périodes estivales et principalement lors des épisodes pluvieux ou bien lorsque le Rhône est en crue, les demandes en eau pour l'irrigation sont restreintes. Les flux captés à l'entrée du canal sont amenuisés et ne correspondent pas directement aux flux d'eau et de matière en transit dans le fleuve que ce soit du point du vue quantitatif (proportionnalité) ou qualitatif (ségrégation des particules). Ce contexte hydraulique limite d'autant plus l'intrusion des matériaux les plus grossiers qui contribuent à la dilution apparente des concentrations massiques dans le fleuve.

\subsection{Résultats des mesures réalisées en 2011 dans le canal géré par BRL}

\subsubsection{Les sédiments}

L'ensemble des résultats d'analyses est présenté dans le tableau 2.

\section{Granulométrie, matière organique et carbone organique}

Les sédiments prélevés au sein du canal sont des matériaux fins caractérisés par des proportions extrêmement faibles de sables fins (inférieures ou égales à $4 \%$ ) et une quasi absence de sables grossiers pour ce qui est de la partie terminale du canal $(<3 \%)$. Ces observations sont à mettre directement en relation avec le fonctionnement hydrodynamique du système. À la Méjanelle, les dépôts sédimentaires sont constitués à près de $94 \%$ de limons et d'argiles, dont plus de $55 \%$ de limons fins. Les sédiments accumulés au niveau de Fourques et de la Méjanelle présentent des similitudes texturales avec 


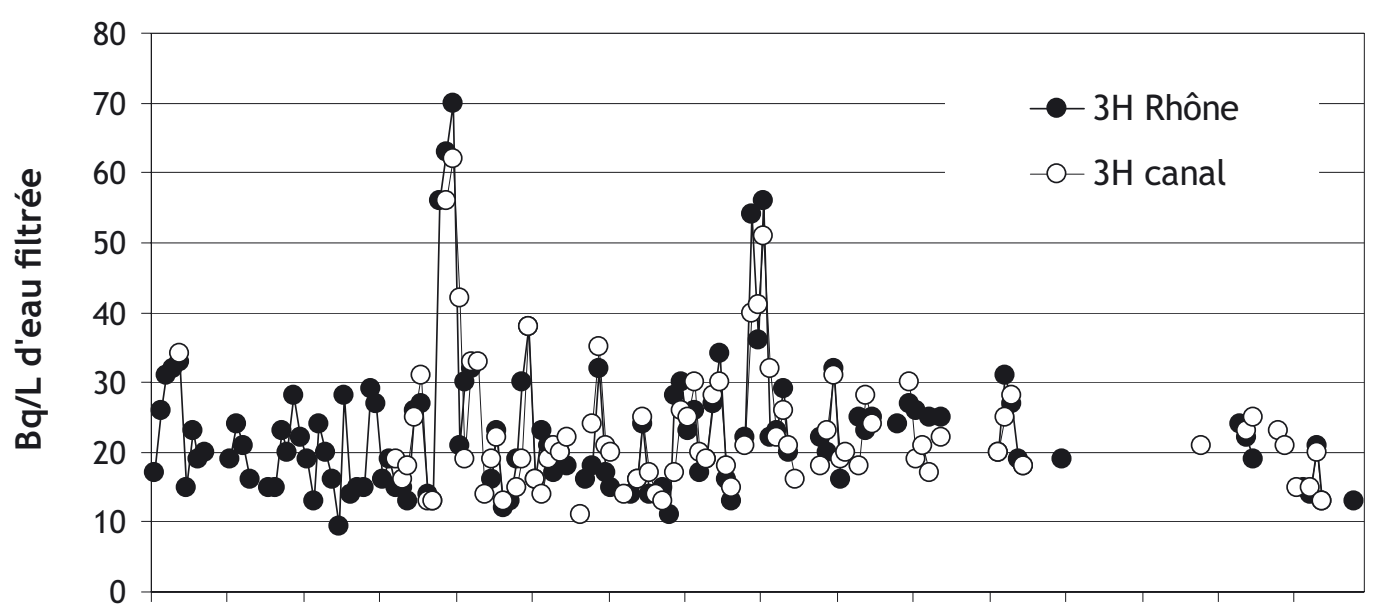

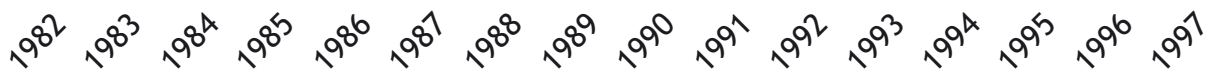

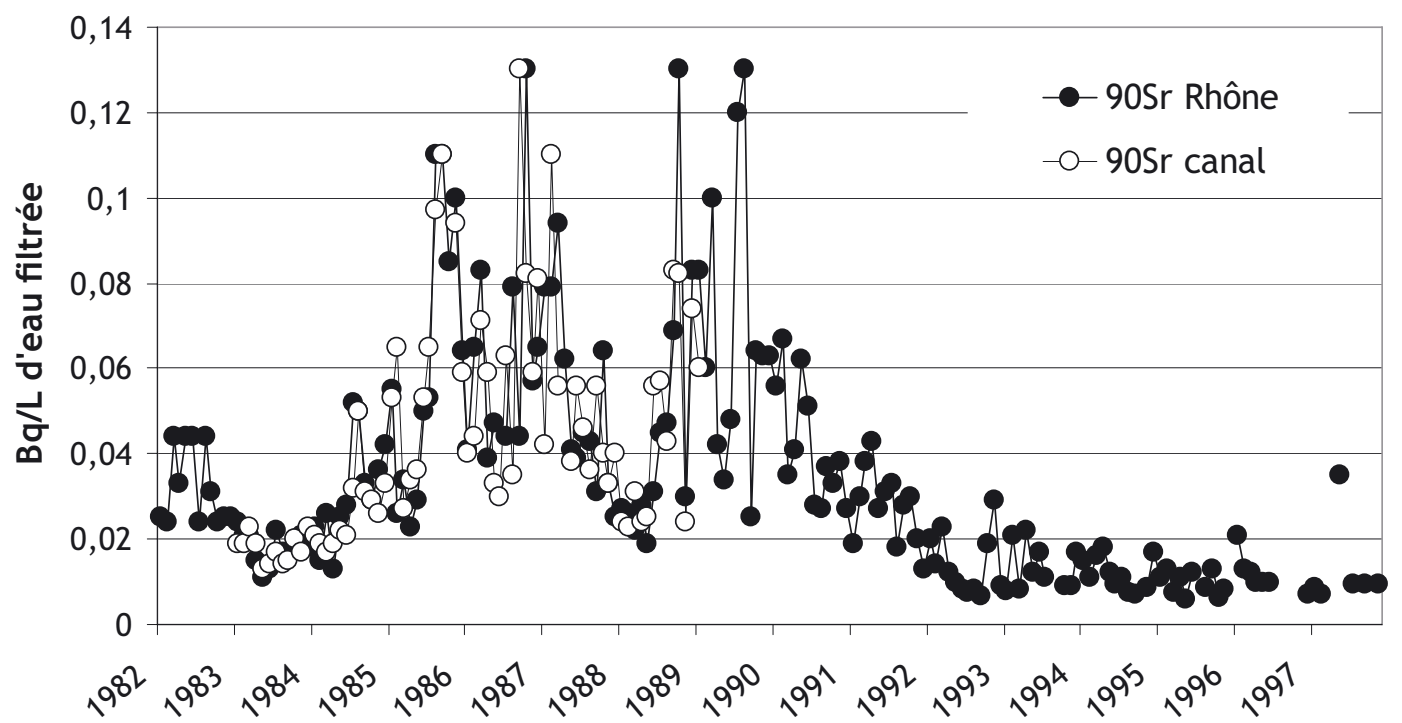

Fig. 2. Activités volumiques en tritium libre $\left({ }^{3} \mathrm{H}\right)$ en haut et en ${ }^{90} \mathrm{Sr}$ en bas, mesurées dans les eaux filtrées du Rhône aval (station de surveillance de Vallabrègues) et du canal (station de pompage A. Dumont).

Activities of tritium $\left({ }^{3} \mathrm{H}\right)$ and ${ }^{90} \mathrm{Sr}$ measured in filtered waters from the Rhône river (monitoring station at Vallabrègues) and the BRL canal (pumping station A. Dumont).

les dépôts des inondations de décembre 2003 (Eyrolle et al., 2006). Les teneurs en matière organique (MO) sont de $2,6 \%$ et $4,9 \%$ pour Fourques et la Méjanelle, respectivement. La plus forte teneur en MO dans les sédiments de la Méjanelle par rapport à l'entrée du canal est liée à une proportion accrue de matériaux fins. Elle peut également être le résultat d'une production biologique autochtone favorisée par les conditions hydrauliques, d'ensoleillement et de température des canaux.

\section{Teneurs en radionucléides}

Comme attendu, l'essentiel de la radioactivité des sédiments du canal est d'origine naturelle. Elle est composée des différents éléments de filiation des chaînes de $1{ }^{238} \mathrm{U}$ et $\mathrm{du}{ }^{232} \mathrm{Th}$ ainsi que du ${ }^{40} \mathrm{~K}$. Les résultats de mesure alpha global et bêta global sont en moyenne de $320 \mathrm{~Bq} / \mathrm{kg}_{\mathrm{sec}}$ et $690 \mathrm{~Bq} / \mathrm{kg}_{\mathrm{sec}}$, respectivement, et s'incluent dans les gammes de valeurs référencées pour les sédiments du Rhône aval (de 150 à $340 \mathrm{~Bq} / \mathrm{kg}_{\text {sec }}$ pour l'alpha global et de 400 à $1700 \mathrm{~Bq} / \mathrm{kg}_{\text {sec }}$ pour le bêta global).

Les seuls radionucléides d'origine artificielle mesurés au sein du compartiment sédimentaire sont, par ordre d'activité moyenne décroissante; les ${ }^{137} \mathrm{Cs}\left(34,9 \mathrm{~Bq} / \mathrm{kg}_{\mathrm{sec}}\right)$, ${ }^{90} \mathrm{Sr}\left(5,0 \mathrm{~Bq} / \mathrm{kg}_{\mathrm{sec}}\right),{ }^{241} \mathrm{Pu}\left(4,2 \mathrm{~Bq} / \mathrm{kg}_{\mathrm{sec}}\right)$, tritium organiquement lié (TOL, 2,5 Bq/ $\left./ \mathrm{kg}_{\mathrm{sec}}\right),{ }^{60} \mathrm{Co}\left(1,2 \mathrm{~Bq} / \mathrm{kg}_{\mathrm{sec}}\right),{ }^{241} \mathrm{Am}$ $\left(0,6 \mathrm{~Bq} / \mathrm{kg}_{\mathrm{sec}}\right),{ }^{239} \mathrm{Pu}\left(0,3 \mathrm{~Bq} / \mathrm{kg}_{\mathrm{sec}}\right),{ }^{240} \mathrm{Pu}\left(0,2 \mathrm{~Bq} / \mathrm{kg}_{\mathrm{sec}}\right)$ et ${ }^{238} \mathrm{Pu}\left(0,1 \mathrm{~Bq} / \mathrm{kg}_{\mathrm{sec}}\right)$. Ces résultats sont globalement en accord avec les observations réalisées pour les sédiments du Rhône aval, en particulier les dépôts des inondations de décembre 2003 présentant des textures similaires (Eyrolle et al., 2006). Les rapports d'activité ${ }^{238} \mathrm{Pu} /{ }^{239+240} \mathrm{Pu}$ sont de $0,19 \pm 0,02$ à Fourques comme à la Méjanelle. Ce rapport d'activité permet d'évaluer que près de $60 \%$ du ${ }^{239+240} \mathrm{Pu}$ et $95 \%$ du ${ }^{238} \mathrm{Pu}$ présents dans les sédiments du canal proviennent des rejets liquides du centre de Marcoule (Rolland et al., 2006; Eyrolle et al., 2008). 

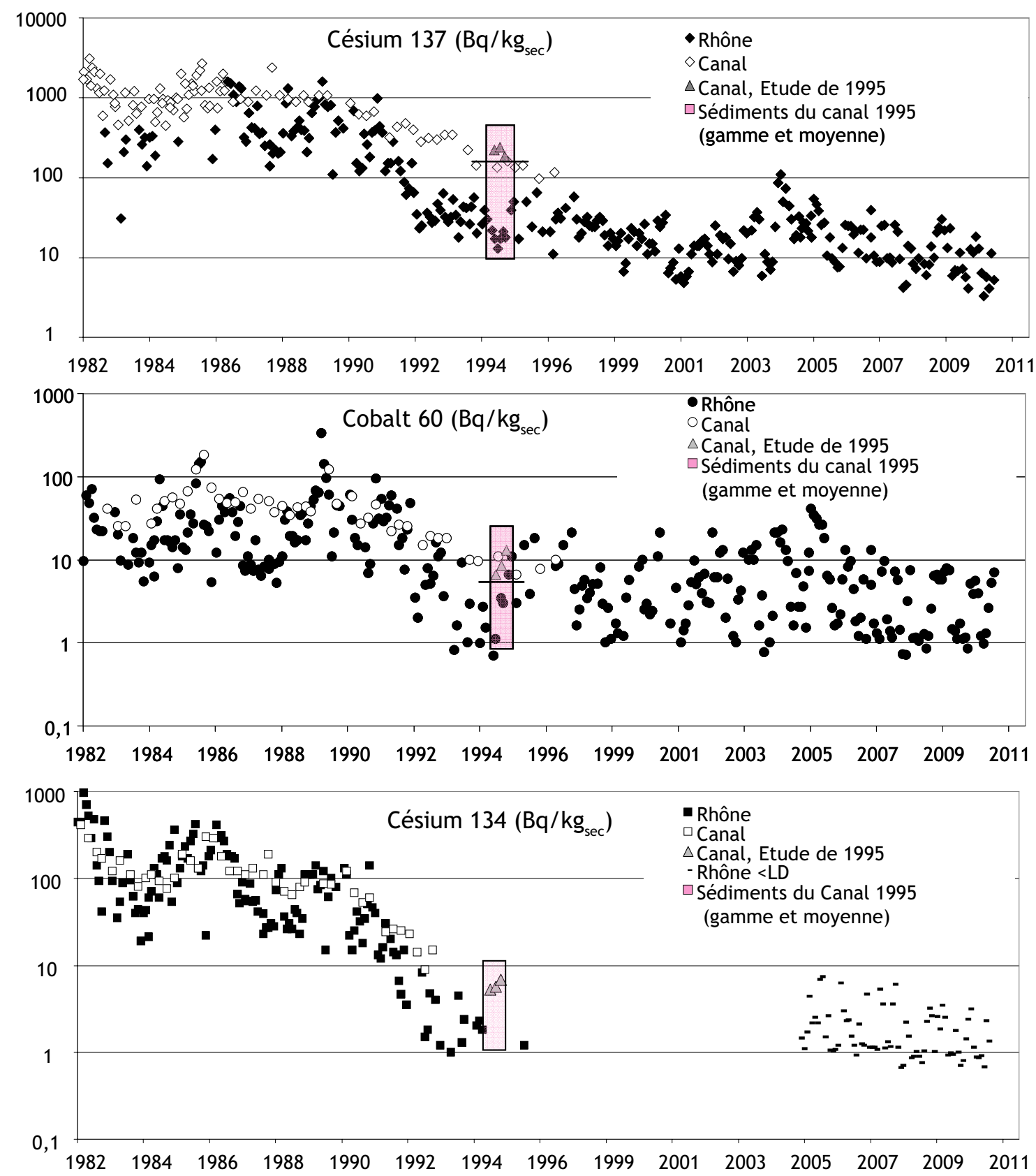

Fig. 3. Séries temporelles des activités massiques en ${ }^{137} \mathrm{Cs},{ }^{60} \mathrm{Co}$ et ${ }^{134} \mathrm{Cs}$ mesurées dans les matières en suspension et les sédiments du Rhône aval et du canal.

Activities of ${ }^{137} \mathrm{Cs},{ }^{60} \mathrm{Co}$ and ${ }^{134} \mathrm{Cs}$ measured in suspended particulate matter and sediments collected in the Rhône river and the canal.

Les teneurs en tritium organiquement lié dans les sédiments du canal varient de 43,5 $\pm 2,2 \mathrm{~Bq} / \mathrm{L}$ à $58,7 \pm 3,0 \mathrm{~Bq} / \mathrm{L}$ d'eau de combustion. Ces résultats indiquent un enrichissement apparent d'un facteur 2,5 à 50 par rapport aux valeurs enregistrées pour le tritium libre dans les eaux du Rhône aval (de $1,0 \pm 0,7 \mathrm{~Bq} / \mathrm{L}$ à $19,4 \pm 1,1 \mathrm{~Bq} / \mathrm{L}$ d'eau de combustion, Eyrolle-Boyer et al., 2013). Cet enrichissement apparent pourrait être lié à une production de $\mathrm{MO}$ autochtone (biofilms, micro algues) généralement concomitante aux faibles débits restreignant la dilution des rejets industriels.
Les activités mesurées dans les sédiments de la partie terminale du canal sont supérieures à celles mesurées à l'entrée du système pour les ${ }^{90} \mathrm{Sr}(\times 14),{ }^{137} \mathrm{Cs}(\times 7),{ }^{241} \mathrm{Am}(\times 6)$, et les différents isotopes du plutonium $(\times 5$ à 6$)$ ainsi que pour le TOL $(\times 1,4)$. Ces observations confortent les résultats de l'étude de 1995 (Jourd'Heuil et al., 1997) et s'expliquent par les différences de texture et de composition des sédiments. La normalisation granulométrique des activités en ${ }^{137} \mathrm{Cs}$ indique en effet que ce paramètre expliquerait près de $75 \%$ de l'enrichissement pour ce radionucléide. 
Tableau 2. Résultats d'analyses des sédiments collectés en 2011 au sein du canal géré par BRL. Inc. : incertitude sur le résultat d'analyse. LD : limite de détection. * : $1^{236} \mathrm{U}$ naturel est en très faible abondance; sa détection témoigne généralement d'un apport anthropique.

Activities measured in sediments collected in the BRL canal in 2011. Inc. : uncertainty. LD : limit of detection. * : natural ${ }^{236} \mathrm{U}$ is very low in abundance ; its detection usually reflects an anthropogenic contribution.

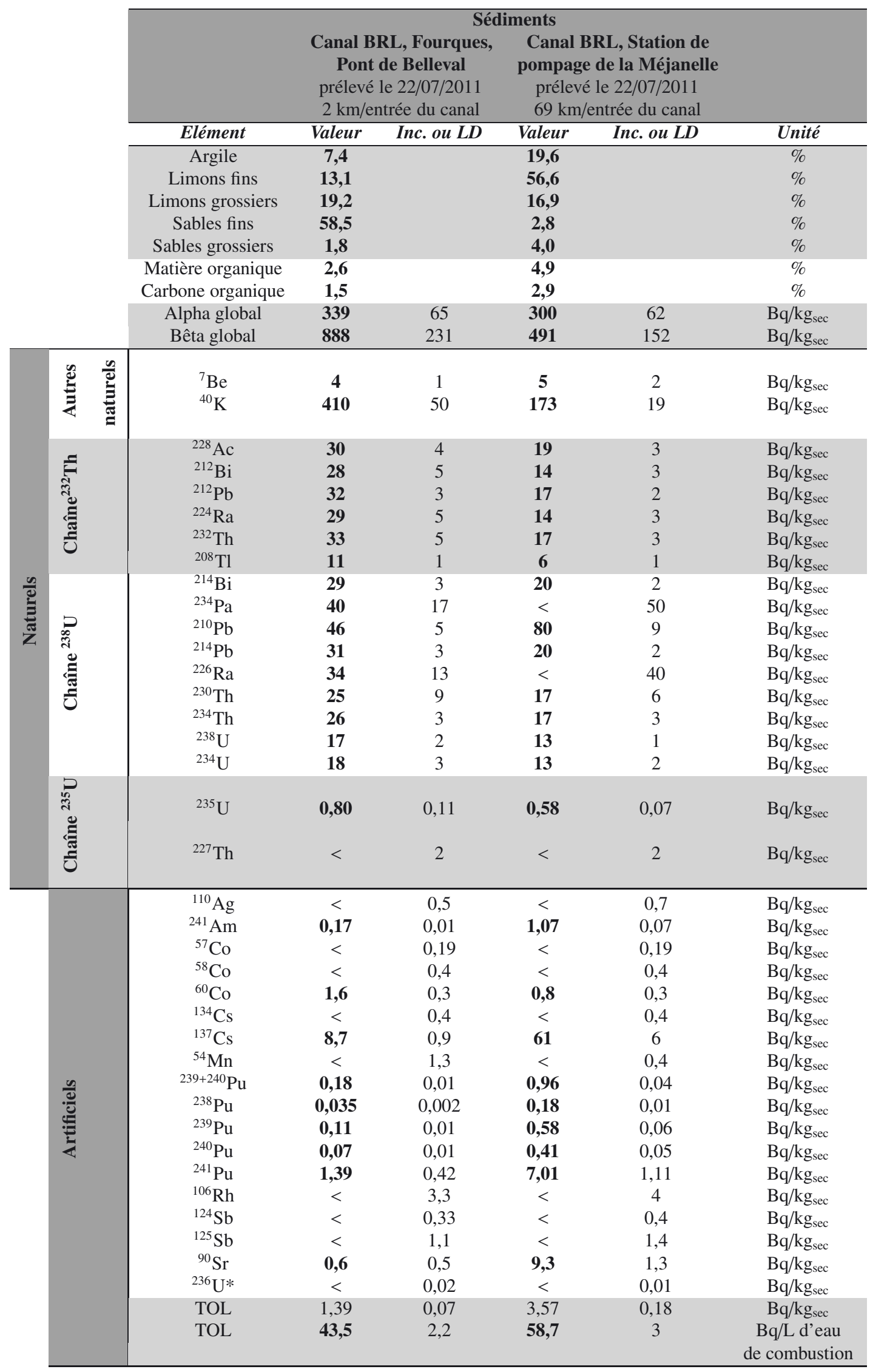


Les différents radionucléides d'origine naturelle mesurés à la Méjanelle présentent $a$ contrario des activités 30 à $50 \%$ plus faibles par rapport à l'entrée du canal, à l'exception du ${ }^{210} \mathrm{~Pb}$. Ces résultats sont à mettre en relation avec les différences texturales des sédiments entre l'entrée et l'aval du système. Les radionucléides naturels d'origine tellurique font partie, outre le ${ }^{40} \mathrm{~K}$, des terres rares et des actinides et composent en grande partie les matrices minérales. Leur partition solide/solution au sein des hydrosystèmes dépend de la nature et de la composition minéralogique des particules (Ramasamy et al., 2011). L'activité massique du ${ }^{40} \mathrm{~K}$ ne peut pas être directement associée à celles $d u^{232} \mathrm{Th}$ et de $1^{1238} \mathrm{U}$ bien que ces éléments soient constitutifs des minéraux présents dans les sols (Megumi et al., 1988). Le ${ }^{40} \mathrm{~K}$ entre en effet principalement dans la composition des minéraux argileux, ainsi, dans un système à l'équilibre, l'activité en ${ }^{40} \mathrm{~K}$ est généralement corrélée avec les teneurs en argiles. Au sein du canal, l'activité du ${ }^{40} \mathrm{~K}$ dans les sédiments et les proportions de particules fines varient en sens inverse. Ces résultats se distinguent des observations généralement reportées dans la littérature et s'expliquent soit par un terme source de ${ }^{40} \mathrm{~K}$ d'origine anthropique, en particulier des apports de fertilisants sur le bassin rhodanien (Barišić et al., 1992 ; Martinez-Aguire et al., 1994 ; Mortvedt, 1994), soit par une dilution de l'activité massique avec la MO produite au sein du canal.

Le ${ }^{210} \mathrm{~Pb}$ est un élément particulièrement réactif vis-à-vis des particules. Cette propriété explique les activités plus élevées mesurées pour cet élément dans les sédiments fins et riches en MO à l'aval du système.

Ces résultats témoignent de l'importance des termes sources ainsi que de la qualité et de l'origine des phases porteuses dans les processus de transfert des différents radionucléides.

\subsubsection{Les matières en suspension}

L'ensemble des résultats d'analyses sont présentés dans le tableau 3 .

\section{Teneurs en matière organique}

Les teneurs en MO mesurées dans les MES du canal sont dans les gammes de valeurs mesurées dans le Rhône (entre 2 et $16 \%$ ) pour les prélèvements réalisés au niveau de la prise de Bouillargues $(4,7 \%)$ et de la station de pompage de la Méjanelle $(14,0 \%)$ en juillet 2011. Par contre, la teneur en MO des MES collectées en décembre 2011 à la Méjanelle est très supérieure aux valeurs généralement enregistrées pour le Rhône aval $(62,4 \%)$. D’une manière générale, il apparaît qu'à l'instar des teneurs en MO dans les sédiments, les teneurs en MO des MES augmentent avec l'éloignement par rapport à l'entrée du canal.

Ces résultats sont à mettre en relation avec une production biologique autochtone (phyto et zooplancton) plus ou moins marquée en fonction de la variation des paramètres physicochimiques tels que la température, la turbidité, la teneur en nutriments, et les conditions d'écoulement et de renouvellement de l'eau. Les parties des canaux les plus éloignées de la prise d'eau au Rhône constituent dans ce cadre des zones propices au développement biologique en raison d'une plus faible charge en suspension, d'un réchauffement éventuel des eaux lié à la diminution de la hauteur d'eau et d'une restriction des vitesses d'écoulement. Par ailleurs, si le mois de juillet peut paraître plus propice aux 'blooms' phyto-planctoniques, il correspond également aux périodes de fortes demandes en eau pour l'irrigation favorisant les écoulements et le renouvellement de l'eau au sein du canal. Les résultats montrent que c'est au début du mois de décembre 2011, caractérisé par une absence de forts débits dans le fleuve (faible charge), une demande en eau à des fins d'irrigation restreinte et des températures clémentes, que les plus fortes teneurs en MO ont été observées. Cette production biologique peut influer de manière significative sur la charge et sur la qualité des matières en suspension, et par conséquent sur la fixation des radionucléides.

\section{Teneurs en radionucléides}

Comme pour les sédiments, la radioactivité des MES du canal est en grande majorité d'origine naturelle. Les activités en ${ }^{7} \mathrm{Be}$, élément d'origine atmosphérique, sont proches de celles mesurées dans les eaux du Rhône hors crue (50 à $300 \mathrm{~Bq} / \mathrm{kg}_{\mathrm{sec}}$ ) et soulignent le transit rapide des masses d'eau. D'une manière générale, les niveaux de radioactivité naturelle mesurés dans les MES du canal sont dans les gammes de valeurs enregistrées pour les MES du Rhône (Antonelli, 2011; Eyrolle et al., 2012). Les activités mesurées dans les MES au niveau de la station de pompage de la Méjanelle sont sensiblement inférieures à celles collectées le jour même à Bouillargues, d'un facteur 0,6 à 0,8 , à l'exception du ${ }^{7} \mathrm{Be}$ $(\times 1,9)$ et ${ }^{210} \mathrm{~Pb}(\times 2,2)$. Ces écarts s'expliquent par les différences de teneur et/ou de qualité de la MO. Ils s'expliquent également très probablement par des différences de la granulométrie des MES (non étudié).

Comparées aux teneurs dans les sédiments, les teneurs dans les MES prélevées en juillet 2011, sont supérieures, d'un facteur 1,1 à 1,9 . Les écarts les plus importants concernent les ${ }^{40} \mathrm{~K},{ }^{224} \mathrm{Ra}$ et ${ }^{210} \mathrm{~Pb}$. A contrario, les teneurs dans les MES prélevées en décembre 2011, présentant une proportion importante de MO, sont moindres que celles des sédiments, d'un facteur 0,5 à 0,8 à l'exception du ${ }^{40} \mathrm{~K}(\times 1,4)$. Ces écarts s'expliquent par des apports différenciés de radioactivité naturelle en fonction de l'origine des masses d'eau, ou bien par la variabilité de la taille des particules et de leur teneur et qualité en MO.

Les radionucléides d'origine artificielle détectés au sein des MES sont, par ordre d'activité moyenne décroissante, les ${ }^{137} \mathrm{Cs}\left(43,3 \mathrm{~Bq} / \mathrm{kg}_{\mathrm{sec}}\right),{ }^{90} \mathrm{Sr}\left(2,1 \mathrm{~Bq} / \mathrm{kg}_{\mathrm{sec}}\right),{ }^{241} \mathrm{Am}$ et ${ }^{60} \mathrm{Co}$ $\left(0,7 \mathrm{~Bq} / \mathrm{kg}_{\mathrm{sec}}\right),{ }^{239+240} \mathrm{Pu}\left(0,5 \mathrm{~Bq} / \mathrm{kg}_{\mathrm{sec}}\right)$ et ${ }^{238} \mathrm{Pu}\left(0,1 \mathrm{~Bq} / \mathrm{kg}_{\mathrm{sec}}\right)$. Ces valeurs sont systématiquement supérieures aux valeurs moyennes enregistrées dans les eaux du Rhône à Arles (SORA), à l'exception près du ${ }^{60} \mathrm{Co}$ (Tab. 4).

La comparaison entre les activités mesurées dans les MES collectées à la station de pompage de la Méjanelle et la prise de Bouillargues montre que la partie terminale du canal présente des activités environ 3 à 5 fois plus élevées. Les écarts les plus importants sont observés pour le ${ }^{137} \mathrm{Cs}(\times 4,8)$ et le ${ }^{90} \mathrm{Sr}(\times 4,4)$. Comme dans le cas des sédiments, si les proportions croissantes de MO et de matériaux fins semblent conduire à une 
Tableau 3. Résultats d'analyses des MES collectées au sein du canal géré par BRL en 2011. Inc. : incertitude sur le résultat de mesure. $\mathrm{LD}$ : limite de détection. $\mathrm{nm}$ : non mesuré. ${ }^{*}: 1^{1236} \mathrm{U}$ naturel est en très faible abondance; sa détection témoigne généralement d'un apport anthropique.

Activities measured in suspended particulate matter collected in the BRL channel in 2011. Inc. : uncertainty. LD : limit of detection. nm : non measured. * : natural ${ }^{236} \mathrm{U}$ is very low in abundance ; its detection usually reflects an anthropogenic contribution.

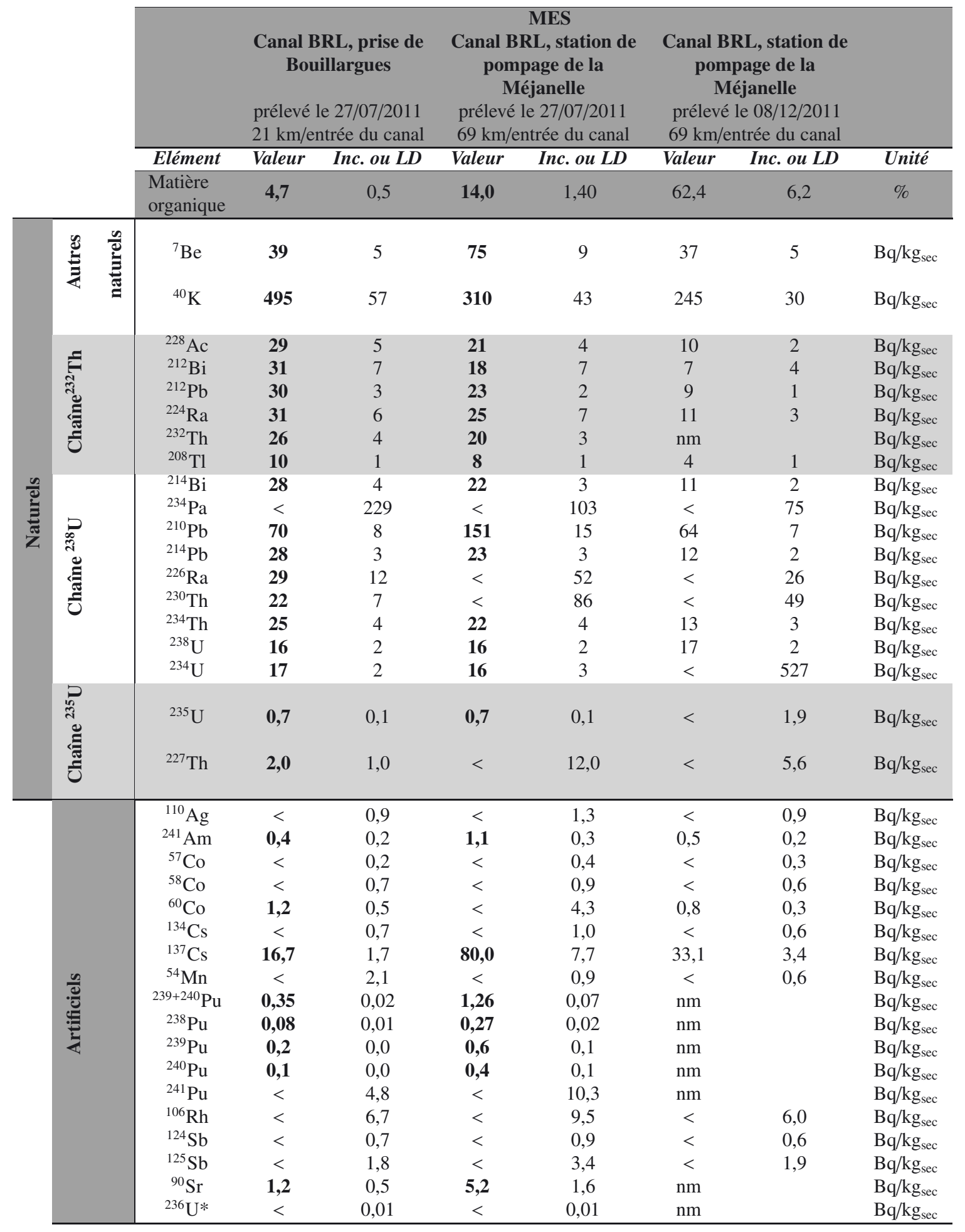




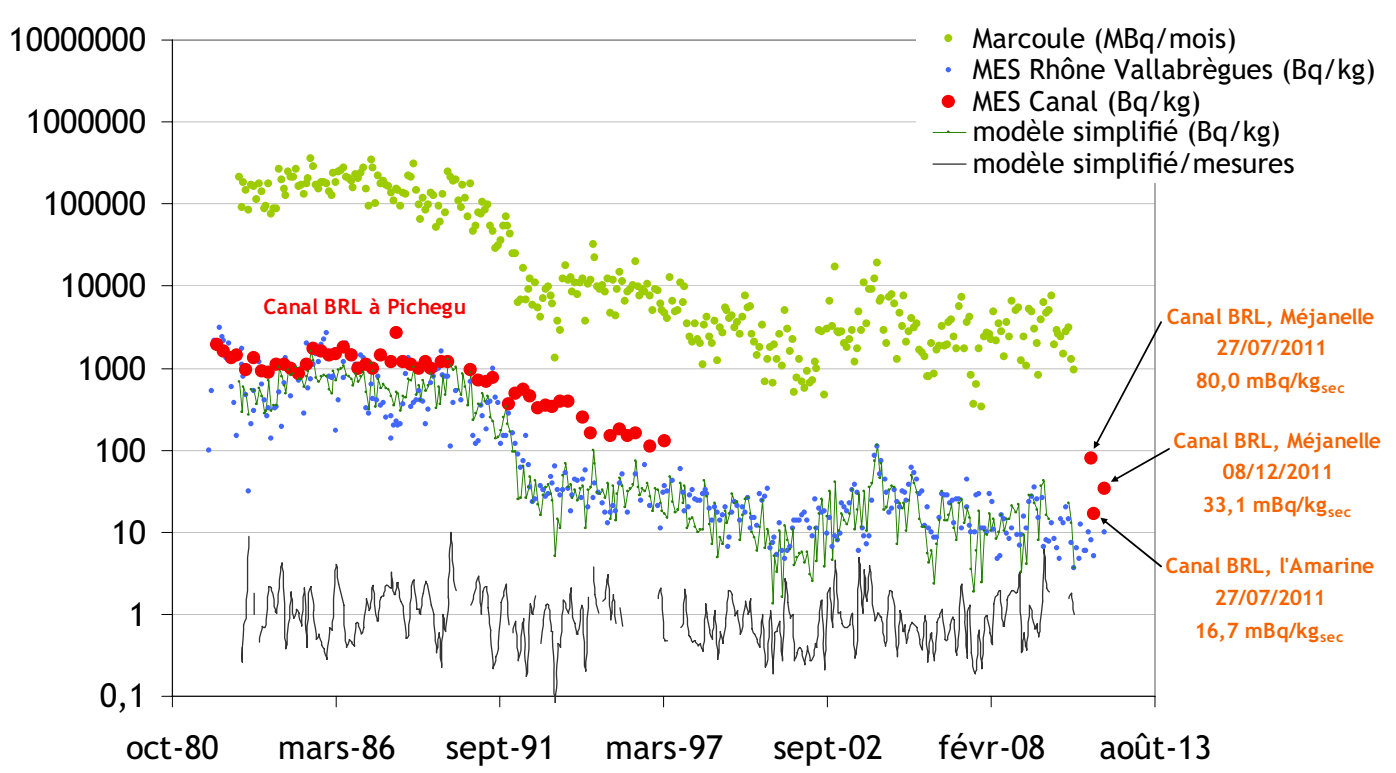

Fig. 4. Chroniques des rejets liquides en ${ }^{137} \mathrm{Cs}$ de Marcoule, des activités en ${ }^{137} \mathrm{Cs}$ des MES mesurées dans le Rhône à Vallabrègues et dans le canal. Activités théoriques attendues dans le Rhône (modèle simplifié) et rapport activités calculées / activités mesurées (modèle simplifié/mesures).

Measurements of ${ }^{137} \mathrm{Cs}$ released by Marcoule spent fuel reprocessing plant, ${ }^{137} \mathrm{Cs}$ activities measured in suspended particulate matter collected in the Rhone river at Vallabrègues and in the canal (data). Theoretical activities (simplified model) and ratios of theoretical activities and measured activities (simplified model/data).

Tableau 4. Gammes d'activités mesurées dans les MES en Arles (SORA) entre 2006 et 2011 (en Bq/ $\mathrm{kg}_{\mathrm{sec}}$ ) pour les éléments d'origine artificielle détectés dans les MES du canal en 2011.

Activities measured in suspended particulate matter collected in the Rhone river at Arles (SORA monitoring station) between 2006 and 2011 (in $\mathrm{Bq} / \mathrm{kg}_{\text {dry }}$ ) for artificial radionuclides detected in the canal in 2011.

\begin{tabular}{lcccc}
\hline & Min. & Max. & Moy. & Ecart-type \\
\hline${ }^{\mathbf{1 3 7}} \mathbf{C s}$ & 3,66 & 49,78 & 12,92 & 8,05 \\
${ }^{\mathbf{9}} \mathbf{S r}$ & 0,47 & 1,64 & 0,95 & 0,32 \\
${ }^{{ }^{60}} \mathbf{C o}$ & 0,24 & 19,91 & 3,57 & 4,19 \\
${ }^{\mathbf{2 4 1}} \mathbf{A m}$ & 0,04 & 1,75 & 0,26 & 0,32 \\
${ }^{\mathbf{2 3 9}+\mathbf{2 4 0}} \mathbf{P u}$ & 0,06 & 0,75 & 0,19 & 0,10 \\
${ }^{\mathbf{2 3 9}} \mathbf{P u}$ & 0,03 & 0,52 & 0,16 & 0,14 \\
${ }^{\mathbf{2 4 0}} \mathbf{P u}$ & 0,02 & 0,48 & 0,13 & 0,13 \\
${ }^{238} \mathbf{P u}$ & 0,004 & 0,28 & 0,03 & 0,04 \\
\hline
\end{tabular}

dilution des activités de la majorité des radionucléides d'origine naturelle, elles conduisent a contrario à une augmentation des teneurs en radionucléides d'origine artificielle. Les mécanismes mis en jeu incluent des processus d'échanges physicochimiques et biologiques, en particulier l'assimilation par le phyto et zooplancton.

Les activités mesurées dans les MES du canal sont supérieures aux valeurs maximales enregistrées dans le Rhône dans le cas du ${ }^{137} \mathrm{Cs}(\times 1,6)$, du ${ }^{90} \mathrm{Sr}(\times 3,2)$ et du ${ }^{239+240} \mathrm{Pu}(\times 1,7)$. Elles sont de l'ordre de grandeur des valeurs maximales mesurées pour les autres radionucléides artificiels détectés à l'exception du ${ }^{60}$ Co pour lequel les activités mesurées sont proches des limites de détection (Tab. 4).
La figure 4 souligne les différences entre les activités en ${ }^{137}$ Cs mesurées dans les MES du Rhône et dans celles du canal. Elle présente également les activités théoriques en ${ }^{137} \mathrm{Cs}$ des MES attendues dans le fleuve, calculées à partir de la connaissance des rejets liquides du centre de retraitement et des débits du fleuve ${ }^{1}$. Les résultats soulignent que les différences d'activités en ${ }^{137} \mathrm{Cs}$ entre les MES de l'eau du canal et celles du fleuve ont été systématiques au cours des dernières décennies, et indépendantes des niveaux de rejets dans le fleuve. Ils indiquent également que le terme source prépondérant de ${ }^{137} \mathrm{Cs}$ dans le Rhône aval a été constitué, comme attendu, par les rejets liquides de Marcoule. En effet, le rapport des activités théoriques et des activités mesurées est resté proche de 1 $(1,16 \pm 1,07)$ sur les 30 dernières années d'enregistrement de mesures.

\subsubsection{L'eau filtrée}

L'ensemble des résultats d'analyses est présenté dans le tableau 5.

La radioactivité observée dans l'eau filtrée du canal est, comme pour les sédiments et les MES, essentiellement d'origine naturelle. Les seuls éléments de filiation des chaînes U-Th

${ }^{1}$ L'activité en ${ }^{137} \mathrm{Cs}$ des $\operatorname{MES}\left(A\right.$, en $\mathrm{Bq} / \mathrm{kg}_{\mathrm{sec}}$ ) est approchée par la relation (1) ci-dessous, avec $M$, les rejets liquides de Marcoule (en $\mathrm{Bq} / \mathrm{s}), Q$ le débit moyen mensuel en $\mathrm{m}^{3} / \mathrm{s}$, MES la concentration en MES en $\mathrm{mg} / \mathrm{L}$ et $\%_{\text {MES }}$ la proportion de ${ }^{137} \mathrm{Cs}$ fixée sur les particules. Nous avons considéré dans cette étude une charge moyenne en MES de $50 \mathrm{mg} / \mathrm{L}$ et $85 \%$ de l'activité associée aux particules.

$$
A=\frac{M}{M E S \times Q} \times \%_{\mathrm{MES}}
$$


Tableau 5. Résultats d'analyses de l'eau filtrée collectée au sein du canal géré par BRL à la Méjanelle en 2011. Inc. : incertitude sur le résultat de mesure. LD : limite de détection. $\mathrm{nm}$ : non mesuré. $*:{ }^{1}{ }^{236} \mathrm{U}$ naturel est en très faible abondance ; sa détection témoigne généralement d'un apport anthropique.

Activities measured in filtered waters collected in the BRL at La Méjanelle in 2011. Inc. : uncertainty. LD : limit of detection. nm : non measured. * : natural ${ }^{236} \mathrm{U}$ is very low in abundance ; its detection usually reflects an anthropogenic contribution.

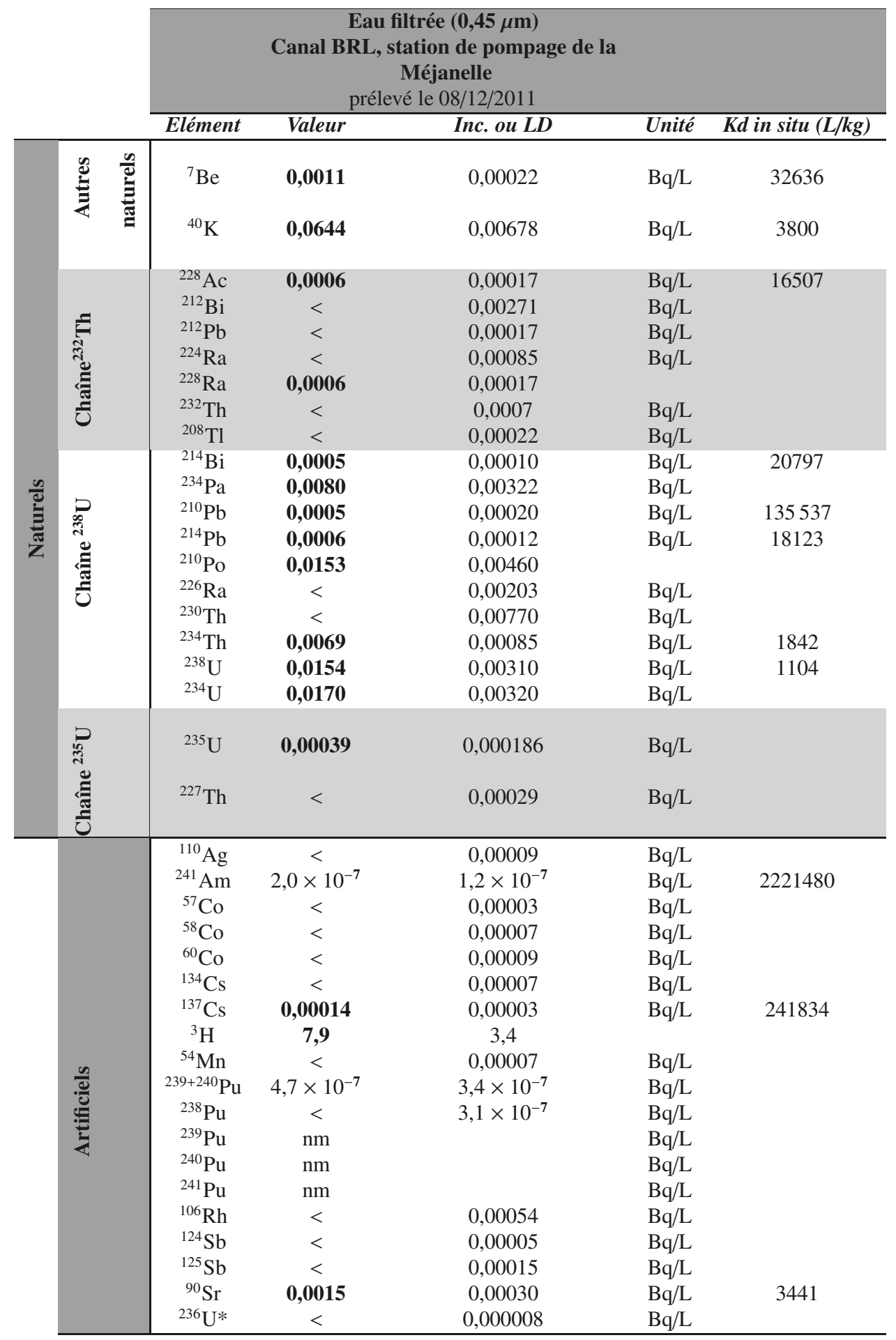




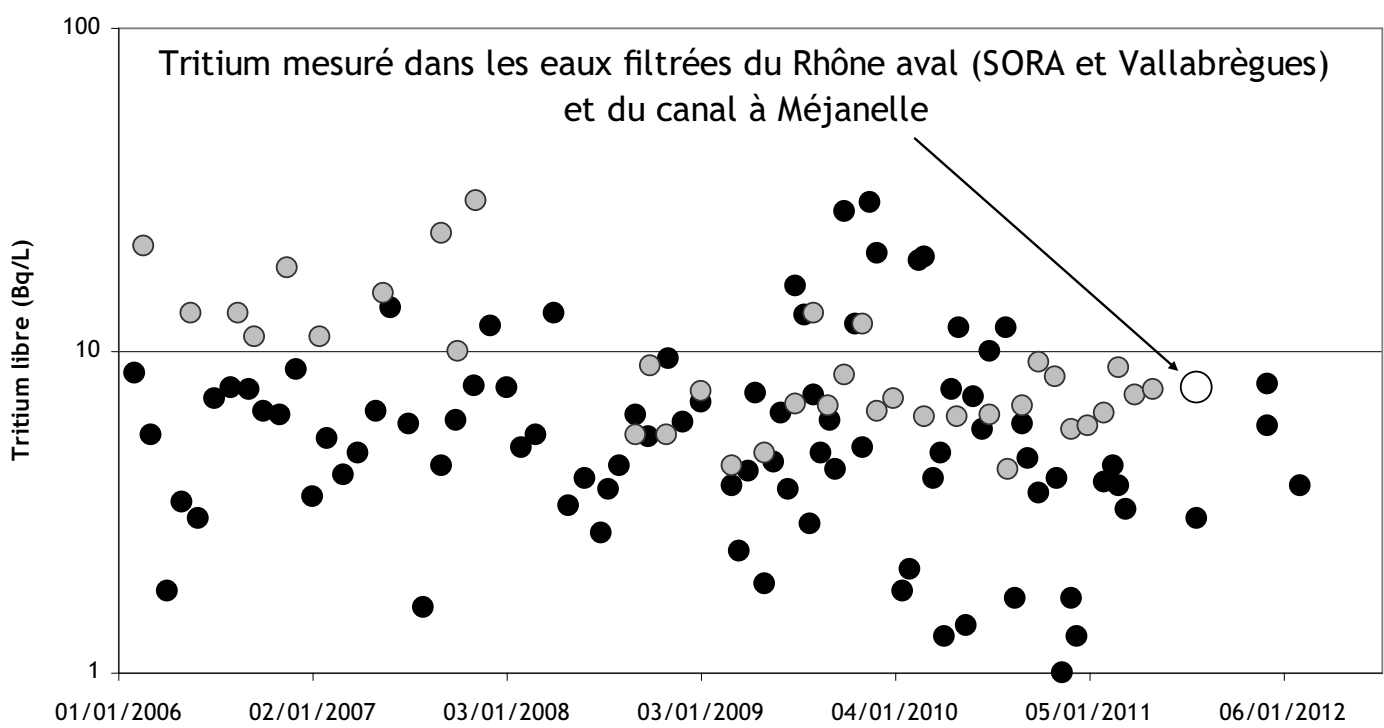

Fig. 5. Concentrations en tritium libre en $\mathrm{Bq} / \mathrm{L}$ dans les eaux filtrées du système rhodanien en aval de Marcoule, à SORA (points noirs), Vallabrègues (points gris) et à Méjanelle (point blanc)

Tritium activities in $\mathrm{Bq} / \mathrm{L}$ measured in filtered waters collected in the Rhone waters downstream of the Marcoule reprocessing plant at Arles (SORA station; black circles), at Vallabrègues (gray circles) and in the BRL canal at La Méjanelle (white circle) from 2006-2012.

détectés dans les eaux filtrées sont, par ordre d'activité décroissante, les ${ }^{234} \mathrm{U},{ }^{238} \mathrm{U},{ }^{210} \mathrm{Po},{ }^{234} \mathrm{~Pa},{ }^{234} \mathrm{Th},{ }^{214} \mathrm{~Pb},{ }^{228} \mathrm{Ac}$ et ${ }^{228} \mathrm{Ra}$, ${ }^{214} \mathrm{Bi},{ }^{210} \mathrm{~Pb}$ et ${ }^{235} \mathrm{U}$. Le ${ }^{40} \mathrm{~K}$ présente l'activité volumique la plus importante $(0,06 \mathrm{~Bq} / \mathrm{L})$. Les éléments détectés au sein de la chaîne de $1{ }^{238} \mathrm{U}$ présentent des déséquilibres significatifs $\left({ }^{238} \mathrm{U} /{ }^{234} \mathrm{Th}=2,2 ;{ }^{234} \mathrm{Th} /{ }^{214} \mathrm{Bi}=13,2\right)$ qui s'expliquent par les différences de comportement physico-chimique des éléments. En effet, les valeurs de $\mathrm{Kd}$ in situ varient sur plusieurs ordres de grandeur (Tab. 5). Les niveaux de radioactivité naturelle mesurés dans l'eau filtrée du canal sont, comme attendu, dans les gammes des valeurs enregistrées dans les eaux filtrées du Rhône (Antonelli, 2011).

Parmi les radionucléides artificiels, le ${ }^{3} \mathrm{H}$ présente l'activité volumique la plus élevée $(7,9 \pm 3,4 \mathrm{~Bq} / \mathrm{L})$, supérieure de 4 à 7 ordres de grandeur à celles des autres radionucléides artificiels. Cette valeur est proche des activités mesurées dans le cadre de la surveillance des eaux du Rhône que ce soit au niveau de Vallabrègues ou de SORA (Fig. 5). Les autres radionucléides d'origine artificielle détectés dans l'eau filtrée collectée à la Méjanelle sont, par ordre d'activité moyenne décroissante, les ${ }^{90} \mathrm{Sr}(1,5 \mathrm{mBq} / \mathrm{L}),{ }^{137} \mathrm{Cs}(0,14 \mathrm{mBq} / \mathrm{L}),{ }^{241} \mathrm{Am}$ et ${ }^{239+240} \mathrm{Pu}(\mathrm{de}$ l'ordre de quelques $0,0001 \mathrm{mBq} / \mathrm{L})$. Comme attendu, ces activités volumiques sont similaires à celles mesurées dans les eaux filtrées du Rhône aval, en particulier par la station SORA (Tab. 6 et Fig. 5).

\section{Conclusions}

Les canaux sont des hydrosystèmes anthropisés aux caractéristiques propres gouvernées par des conditions hydrologiques atypiques. Ces conditions peuvent influencer les processus de transfert et le devenir des contaminants classiquement observés au sein des hydrosytèmes naturels. Les radionucléides détectés actuellement dans les eaux du canal
Tableau 6. Gammes d'activités mesurées dans les eaux filtrées à Arles (SORA) entre 2006 et 2011 (en $\mathrm{Bq} / \mathrm{L}$ ) pour les éléments d'origine artificielle détectés dans l'eau filtrée du canal en 2011.

Activities measured in filtered waters collected in the Rhone river at Arles (SORA monitoring station) between 2006 and 2011 (in Bq/L) for artificial radionuclides detected in the filtered waters from the canal in 2011 .

\begin{tabular}{lcccc} 
& Min. & Max. & Moy. & Ecart-type \\
\hline${ }^{\mathbf{1 3 7}} \mathbf{C s}$ & 0,00009 & 0,00095 & 0,00036 & 0,00021 \\
${ }^{\mathbf{9}} \mathbf{S r}$ & 0,00051 & 0,00335 & 0,00172 & 0,00080 \\
${ }^{241} \mathbf{A m}$ & 0,0000002 & 0,0000080 & 0,0000015 & 0,0000016 \\
${ }^{239+240} \mathbf{P u}$ & 0,0000002 & 0,0000070 & 0,0000018 & 0,0000014 \\
Tritium & 1,00 & 61,50 & 7,61 & 8,19 \\
\hline
\end{tabular}

Philippe Lamour, propriété de la région Languedoc Roussillon géré par BRL, sont en grande majorité d'origine naturelle et concernent les éléments de filiation des chaînes (U-Th) et le ${ }^{40} \mathrm{~K}$. Nos résultats ont permis de montrer que le suivi des niveaux de radioactivité des eaux du Rhône permet d'avoir une bonne représentation des activités en transit au sein du canal, en particulier au sein de la phase dissoute. Les activités en radionucléides artificiels au sein des MES et sédiments sont plus importantes dans la partie terminale du canal par rapport à l'entrée (prise d'eau au Rhône). A contrario, les activités des différents éléments de filiation des chaînes U-Th ainsi que le ${ }^{40} \mathrm{~K}$ diminuent de 30 à $50 \%$, dans la plupart des cas. Ces observations s'expliquent par les différences de texture et de composition des matières solides entre la partie amont du canal et sa partie terminale.

Nos résultats soulignent la ségrégation des particules selon un gradient longitudinal généré par la décantation préférentielle des matériaux les plus grossiers en amont du système et un enrichissement apparent en particules fines vers l'aval. Ils 
montrent également que les teneurs en MO des MES et des sédiments du canal sont plus importantes que celles enregistrées pour des matrices similaires dans le fleuve, et qu'elles augmentent de l'amont vers l'aval. La production primaire favorisée au sein de ces systèmes est très probablement à l'origine de ces observations, et pourrait expliquer pour partie les teneurs relativement élevées en certains radionucléides $\left({ }^{210} \mathrm{~Pb}\right.$ et tritium organiquement lié).

Dans ces milieux dulçaquicoles particuliers, il semble que les processus biologiques puissent jouer un rôle non négligeable dans le transfert de certains éléments potentiellement contaminants, en particulier le ${ }^{210} \mathrm{~Pb}, 1 \mathrm{e}^{210} \mathrm{Po}$ et le tritium vers les matières en suspension, et vers la chaîne trophique aquatique. Ces mécanismes sont toutefois sans incidence sur la qualité des eaux filtrées.

Remerciements. Les auteurs tiennent à remercier l'Agence de l'Eau Rhône Méditerranée Corse pour son soutien financier de la Station SORA ainsi que l'ensemble des partenaires de l'Observatoire des Sédiments du Rhône de la Zone Atelier Bassin du Rhône.

\section{Références}

Antonelli C. (2011) Flux de radioactivité exportés par le Rhône en Méditerranée en 2009, Rapport IRSN/DEI/SESURE/2011-25.

Antonelli C., Eyrolle F., Boullier V., Gurriaran R., Cossonnet C. (2012) Measurements, quantification and budget recoveries of radionuclide fluxes exported by Rhône River to the Mediterranean Sea, Mesures, estimation et bilan des flux de radioactivité exportés par le Rhône en Méditerranée, I.S. Rivers, 1ère Conference Internationale, 26-28 juin 2012, Lyon, France.

Barišić D., Lulić S., Miletić P. (1992) Radium and uranium in phosphate fertilizers and their impact on the radioactivity of waters, Water Research 26 (5), 607-611.

Eyrolle F., Duffa C., Rolland B., Antonelli C., Leprieur F. (2006) Radiological consequences of the extreme flooding of the Rhône valley area (South east France, December 2003), Sci. Total Environ. 366, 427-438.

Eyrolle F., Claval D., Gontier G., Antonelli C. (2008) Radioactivity level in major French rivers: summary of monitoring chronicles acquired over the past thirty years and current status, J. Environ. Monit. 10, 800-811.
Eyrolle F., Antonelli C., Raimbault P., Boullier V., Arnaud M. (2010) SORA: a high frequency flux monitoting station at the lower Rhône River. In: proceedings of the 39th CIESM Congress, 10-14 May 2010, Venice, Italy.

Eyrolle F., Radakovitch O., Raimbault P., Charmasson C., Antonelli C., Ferrand E., Raccasi G., Aubert D., Gurriaran R. (2012) Consequences of hydrological events on suspended sediment and associated radionuclide deliveries from the Rhône River towards the Mediterranean Sea, J. Soils Sed. 12, 1479-1495.

Eyrolle-Boyer F., Claval D., Antonelli C., Tournieux D., Cossonnet C. (2013) 1963-2013: Fifty years of anthropogenic tritium in our environment, Focus on the Rhône Valley (South east France). In: proceedings of the 10th International Conference on Tritium Science and Technology "TRITIUM 2013", 21-25 October 2013, Nice Acropolis, France.

Jourd'heuil L., Lambretchts A. (1995) Radioactivité des sédiments, des matières en suspension et des eaux prélevés dans les canaux du Bas-Rhône-Languedoc - Année 1995, Rapport IPSN 97/024.

Jourd'heuil L., Lambretchts A., Morello M. (1997) Radioactivité des sédiments, des matières en suspension et des eaux prélevés dans les canaux du Bas-Rhône-Languedoc - Année 1995 - Synthèse, Rapport IPSN 97/024.

Martínez-Aguirre A., García-León M., Ivanovich M. (1994) The distribution of $\mathrm{U}$, Th and ${ }^{22} \mathrm{R}$ a derived from the phosphate fertilizer industries on an estuarine system in Southwest Spain, J. Environ. Radioact. 22 (2), 5-177.

Megumi K., Oka T., Doi M., Kimura S., Rsujimoto T., Ishiyama T., Katsurayama K. (1988) Relationships between the concentrations of natural radionuclides and the mineral composition of the surface soil, Radiat. Prot. Dosim. 24 (1-4), 69-72.

Mortvedt J.J. (1994) Plant and soils relationships of uranium and thorium decay series radionuclides - A review, J. Environ. Qual. 23, 643-650.

Ramasamy V., Suresh G., Meenakshisundaram V., Ponnusamy V. (2011) Horizontal and vertical characterization of radionuclides and minerals in river sediments, Appl. Radiat. Isotopes 69, 184195.

Rolland B., Eyrolle F., Bourles D. (2006) Transfert des Radionucléides artificiels par voie fluviale : Conséquences sur les stocks sédimentaires et les exports vers la Méditerranée, Radioprotection 41 (2), 6-8.

Cite this article as: F. Eyrolle-Boyer, Ph. Renaud, F. Le Dore, D. Tournieux, D. Claval, J.-F. Blanchet, C. Antonelli, M. Zebracki, C. Cossonnet, B. Boulet, X. Cagnat, A. Devisme, R. Gurriaran. Caractéristiques radiologiques des canaux de transport d'eau - Exemple du réseau hydraulique régional Languedoc Roussillon. Radioprotection 49(2), 123-134 (2014). 